\title{
Coherencia y entrelazamiento en dinámica no Markoviana de qubits
}

\author{
Cristian E. Susa ${ }^{1 \bowtie}$, John H. Reina ${ }^{1 凶}$
}

\section{Coherence and entanglement in non-Markovian dynamics of qubits}

\section{Abstract}

We provide a thorough analysis of the entanglement dynamics of an interacting two-qubit system in the non-Markovian regime. In such a regime, the time scale on which the reservoir degrees of freedom evolve is either of the same order of magnitude or less than that on which the system evolves. We used an exact numerical method; the Quasi-Adiabatic Path Integral (QUAPI) technique, to describe the corresponding qubit dissipative dynamics in such a non-Markovian regime. We computed the time evolution of the density operator for the quibits, from which we quantified the coherences and population dynamics, as well as the qubit-bath coupling effects. Using negativity as a metric, we calculated the dynamics of non-local quantum correlations (entanglement), and indentified a non-Markovian quantum phenomena in terms of early stage disentanglement, and the collapse and revival of entanglement.

Keywords: Qubits; quantum information; entanglement; non-Markovian decoherence; nanostructures.

Edited by Humberto Rafeiro E Alberto Acosta

1 Departamento de Física, Universidad del Valle, A.A. 25360, Cali, Colombia.

Received: 11-02-2013 Accepted: 04-06-2013 Published online: 23-06-2013

Citation: Susa EC, Reina JH (2013) Coherencia y entrelazamiento en dinámica no Markoviana de qubits. Universitas Scientiarum 18(2): 141-152 doi: 10.11144/Javeriana.SC18-2.cedm

Funding: Universidad del Valle; Colciencias.

Electronic supplementary material: N/A

SICI: 2027-1352(201305/08)18:2<141:CYEEDNMDQ>2.0.TS;2-B11

\section{Introducción}

El entrelazamiento cuántico es una propiedad física de sumo interés tanto conceptualmente, como argumentación de nivel primario en la teoría cuántica (Mermin 1985, Bennett y DiVincenzo 2000), como por sus aplicaciones en las tecnologías cuánticas de información emergentes (Ladd et al. 2010). Dicha propiedad sólo puede atribuirse a sistemas interactuantes en el dominio cuántico (Nielsen y Chuang 2000). Para el caso más simple de estados puros en un registrador de dos bits cuánticos (qubits), se dice que el sistema está en estado entrelazado cuando este no puede ser descrito como un producto directo de vectores de estado 
asociados a cada una de las dos partes por separado.

El entrelazamiento ha sido reconocido como un recurso (como lo es la energía) para construir novedosos dispositivos en la nano-escala, donde ahora es posible el procesamiento y la manipulación controlada de información al nivel de pocos qubits (Ladd et al. 2010). El entrelazamiento es probablemente la característica más interesante de la fenomenología cuántica (Mermin 1985), y es un recurso crucial para el cómputo cuántico (Bennett y DiVincenzo 2000), y la comunicación cuántica via protocolos como la teletransportación (Bennett, Brassard, Crépeau, Jozsa, Peres y Wootters 1993) y la criptografía (Gisin, Ribordy, Tittel y Zbinden 2002).

Trabajos recientes sobre la dinámica de entrelazamiento han mostrado que algunos estados inicialmente entrelazados de un sistema bipartito pueden decaer a cero en un tiempo mucho menor que el de su emisión espontánea ( $\mathrm{Yu}$ y Eberly 2003, 2004, 2006, Konrad et al. 2008, Dodd y Halliwell 2004, Minter, Carvalho, Kus y Buchleitner 2005, Quiroga y Rodriguez 2007, Scala, Migliore, Messina y Sánchez-Soto 2011). Este fenómeno, denominado desvanecimiento repentino de entrelazamiento (ESD-early stage disentanglement) es una característica cuántica que señala una dinámica disipativa inusual de correlaciones cuánticas puramente no locales (Mermin 1985, Bennet et al. 1993). Ha sido demostrado también que bajo escenarios específicos es posible generar entrelazamiento de manera repentina (ESB-entanglement sudden birth) (Ficek y Tanaś 2008, Almeida et al. 2007). Estas características del entrelazamiento se han estudiado tanto en regímenes Markovianos (Yu y Eberly 2009, Susa y Reina 2010, Ficek y Tanás 2002), como no Markovianos (Bellomo, Lo Franco y Compagno 2007, Cummings y Hu 2008) en diversos contextos (Yu y Eberly 2009, Bellomo et al. 2007, Thorwart, Eckel, Reina, Nalbach y Weiss 2009, Shresta, Anastopoulos, Dragulescu y $\mathrm{Hu}$ 2005), diferentes del aquí analizado. Recientemente se ha reportado sobre la cuantificación de correlaciones cuánticas más allá del entrelazamiento (Fanchini, Cornelio, de Oliveira y Caldeira 2011), en términos de la dinámica de la discordia cuántica (Ollivier y Zurek 2001), correlaciones que capturan, de manera diferente al entrelazamiento, el grado de cuanticidad de un sistema físico.

Aquí, la dinámica disipativa del entrelazamiento en un sistema bipartito de qubits acoplados en el régimen no-Markoviano es analizada. La técnica numérica exacta denominada método cuasi-adiabático basado en integrales de trayectorias
(Makri y Makarov 1994, 1995) es utilizada, esta técnica ha sido empleada exitosamente en sistemas de fase condensada (Makri y Makarov 1994, 1995) y atómico-moleculares (Eckel et al. 2009, Thorwart et al. 2009), por mencionar algunos pocos.

\section{Formulación del problema}

Hamiltoniano del sistema: Los sistemas de dos qubits interactuantes pueden describirse por un Hamiltoniano de la forma (Hammerer, Vidal y Cirac 2002)

$$
H_{S}=H_{0}+H_{12} \text {, }
$$

donde $H_{0}=H_{1}+H_{2}$ denota el término de partícula libre, $H_{i}=-\frac{\hbar}{2} \omega_{i} \sigma_{z}^{(i)}$ corresponde a la energía de transición asociada al qubit $i, \mathrm{y}$

$$
H_{12}=\hbar\left(J_{x} \sigma_{x}^{(1)} \otimes \sigma_{x}^{(2)}+J_{y} \sigma_{y}^{(1)} \otimes \sigma_{y}^{(2)}+J_{z} \sigma_{z}^{(1)} \otimes \sigma_{z}^{(2)}\right) \text {, }
$$

escrita en términos de las matrices de Pauli, denota una interacción tipo Heisenberg anisotrópica (Patterson y Bailey 2010) entre los qubits, y $\hbar$ es la constante de Planck dividida por $2 \pi$. En procesos físicos, tales como la transferencia resonante de energía en sistemas biomoleculares (Thorwart et al. 2009, Eckel, Reina y Thorwart 2009) o en átomos artificiales interactuantes (Reina, Quiroga y Johnson 2000, Lovett, Reina, Nazir y Briggs 2003), $J_{x}=$ $J_{y} \equiv J_{x y}$, y la razón $J \equiv J_{x y} / J_{z}$ determina el tipo de interacción experimentada por los qubits acoplados y define el tipo de entrelazamiento que puede ser generado en el registrador cuántico (Hammerer et al. 2002).

En lo concerniente a la interacción entre el registrador cuántico y el reservorio o baño, se considera el modelo de un sistema de osciladores armónicos unidimensionales (en términos prácticos un continuo de modos bosónicos) acoplados de manera bi-lineal (Feynman y Vernon 1963, Caldeira y Laggett 1983, Weiss 2008) al sistema de qubits. Dentro de este modelo, el Hamiltoniano total (sistema + baño) se puede escribir como (Weiss 2008)

$$
\begin{aligned}
& H=\frac{P^{2}}{2 M}+V(Q, t)+ \\
& \sum_{j}^{N} \frac{1}{2}\left(\frac{p_{j}^{2}}{m_{j}}+m_{j} \omega_{j}^{2}\left(q_{j}-\frac{g_{j}}{m_{j} \omega_{j}^{2}} Q\right)^{2}\right),
\end{aligned}
$$

donde $\{Q, P\}$ denota la coordenada del sistema y su momento conjugado, respectivamente, y $\left\{q_{j}, p_{j}\right\}$ es la posición y el respectivo momento conjugado del modo $j$-ésimo del baño de osciladores con frecuencia $\omega_{j}, y g_{j}$ es la constante de acoplamiento 
sistema-baño. Toda la información sobre el baño que es de crucial importancia en la evolución del sistema está incorporada en la conocida función de densidad espectral (Weiss 2008)

$$
J(\omega)=\frac{\pi}{2} \sum_{j}^{N} \frac{g_{j}^{2}}{m_{j} \omega_{j}^{2}} \delta\left(\omega-\omega_{j}\right) .
$$

Método de la Integral de Caminos Cuasiadiabática-QUAPI: Con el fín de obtener la evolución temporal del sistema, se procede a introducir el método utilizado para encontrar la matriz densidad reducida del registrador cuántico (en adelante $\hbar=1$ ). Esta se calcula tomando la traza sobre los grados de libertad del reservorio,

$$
\rho(t)=\operatorname{Tr}_{B}\left(e^{-\mathrm{i} H\left(t-t_{0}\right)} \sigma(0) e^{\mathrm{i} H\left(t-t_{0}\right)}\right),
$$

donde el conjunto sistema-baño, cuya matriz densidad se denota por $\sigma(t)$, es considerado un sistema con evolución unitaria. Escribiendo el Hamiltoniano $H=H_{s}+H_{B}$, con

$$
\begin{gathered}
H_{s}=\frac{P^{2}}{2 M}+V(Q, t)+\sum_{j}^{N} \frac{g_{j}^{2}}{m_{j} \omega_{j}^{2}} Q^{2}, \\
H_{B}=\sum_{j}^{N} \frac{1}{2}\left(\frac{p_{j}^{2}}{m_{j}}+m_{j} \omega_{j}^{2} q_{j}\right)+\sum_{j}^{N} \frac{g_{j}}{m_{j} \omega_{j}^{2}} q_{j} Q,
\end{gathered}
$$

se realiza la discretización correspondiente para hacer del método de la integral de caminos (Feynman y Vernon 1963) uno numérico (Makri y Makarov 1994, 1995): el operador evolución $e^{-\mathrm{i} H\left(t-t_{0}\right)}$ es reescrito como $\left(e^{-\mathrm{i} H \delta t}\right)^{M}$. Insertando el conjunto completo de estados propios

$$
\int d Q|Q\rangle\left\langle Q\left|\prod_{j}^{N} \int d q_{j}\right| q_{j}\right\rangle\left\langle q_{j}\right|
$$

entre cada par de operadores $e^{-\mathrm{i} H \delta t}$, y usando una partición adiabática de este operador exponencial

$$
e^{\mathrm{i} H \delta t} \approx e^{\mathrm{i} H_{B} \delta t / 2} e^{\mathrm{i} H H_{s} \delta t} e^{\mathrm{i} H_{B} \delta t / 2}
$$

la matriz densidad reducida puede escribirse, en la representación de posición, como la integral de trayectorias (Makri y Makarov 1994, 1995)

$$
\begin{aligned}
\rho\left(Q^{\prime \prime},\right. & \left.Q^{\prime} ; t=M \delta t\right)=\int d Q_{0}^{+} \ldots d Q_{M-1}^{+} \\
& \times \int d Q_{0}^{-} \ldots d Q_{M-1}^{-} \\
& \times\left\langle Q^{\prime \prime}\left|e^{-i H_{s} \delta t}\right| Q_{M-1}^{+}\right\rangle \ldots \\
& \times\left\langle Q_{0}^{+}|\rho(0)| Q_{0}^{-}\right\rangle \ldots \\
& \times\left\langle Q_{M-1}^{-}\left|e^{\mathrm{i} H_{s} \delta t}\right| Q^{\prime}\right\rangle \\
& \times I\left(Q_{0}^{+}, \ldots, Q^{\prime \prime}, Q_{0}^{-}, \ldots, Q^{\prime} ; \delta t\right)
\end{aligned}
$$

donde cada producto

$$
\left\langle Q_{i}^{+}\left|e^{-\mathrm{i} H_{s} \delta t}\right| Q_{j}^{+}\right\rangle\left\langle Q_{j}^{-}\left|e^{\mathrm{i} H_{s} \delta t}\right| Q_{i}^{-}\right\rangle
$$

identifica el propagador libre. Aquí son consideradas las posibles trayectorias antes $\left(Q_{i}^{-}\right)$y después $\left(Q_{i}^{+}\right)$del tiempo referenciado como cero, y hemos asumido que al inicio de la dinámica $(t=0)$, el sistema estaba desacoplado del baño (Weiss 2008): $\sigma(0)=\rho(0) \otimes \rho_{B}(0)$, donde el baño se asume en equilibrio térmico, $\rho_{B}=\frac{e^{\beta H_{B}}}{Z}$, y $Z$ es la función de partición asociada. Así, $Q^{\prime \prime}=Q_{M}^{+}$y $Q^{\prime}=Q_{M}^{-}$, con

$$
\begin{aligned}
I\left(Q_{0}^{+}, \ldots,\right. & \left.Q^{\prime \prime}, Q_{0}^{-}, \ldots, Q^{\prime} ; \delta t\right) \\
& =\operatorname{Tr}_{B}\left[e^{-\mathrm{i} H_{B}\left(Q^{\prime \prime}\right) \delta t / 2} \ldots\right. \\
& \times e^{-\mathrm{i} H_{B}\left(Q_{0}^{+}\right) \delta t / 2} \rho_{B}(0) e^{\mathrm{i} H_{B}\left(Q_{0}^{-}\right) \delta t / 2} \ldots \\
& \left.\times e^{\mathrm{i} H_{B}\left(Q^{\prime}\right) \delta t / 2}\right],
\end{aligned}
$$

el funcional de influencia que incorpora toda la información del baño que es requerida en la descripción de la dinámica del sistema de qubits. Este funcional puede escribirse como (Makri y Makarov 1994, 1995)

$$
\begin{aligned}
I=\exp \left[-\sum_{k=0}^{M} \sum_{k^{\prime}}^{k}\left(Q_{k}^{+}-Q_{k}^{-}\right)\right. & \\
& \left.\left(\eta_{k k^{\prime}} Q_{k^{\prime}}^{+}-\eta_{k k^{\prime}}^{*} Q_{k^{\prime}}^{-}\right)\right],
\end{aligned}
$$

que no es más que la versión discreta del funcional introducido inicialmente por Feynman y Vernon (1963) para las trayectorias continuas $Q^{ \pm}(t)$ :

$$
\begin{aligned}
& I=\exp \left[-\int_{0}^{t} d t^{\prime} \int_{0}^{t^{\prime}} d t^{\prime \prime}\left(Q^{+}\left(t^{\prime}\right)-Q^{-}\left(t^{\prime}\right)\right)\right. \\
& \left.\times\left(\alpha\left(t^{\prime}-t^{\prime \prime}\right) Q^{+}\left(t^{\prime \prime}\right)-\alpha^{*}\left(t^{\prime}-t^{\prime \prime}\right) Q^{-}\left(t^{\prime \prime}\right)\right)\right],
\end{aligned}
$$


donde

$$
\begin{array}{r}
\alpha(t)=\frac{1}{\pi} \int_{0}^{\infty} d \omega J(\omega)[\operatorname{coth}(\beta \omega / 2) \cos (\omega t) \\
-\mathrm{i} \sin (\omega t)]
\end{array}
$$

es el conocido kernel de memoria o función de autocorrelación, el cual determina la no localidad del funcional de influencia. En los casos donde la aproximación de Markov (Gardiner y Zoller 2004) es suficientemente buena para describir la dinámica del registrador, la parte real de la función (2) es proporcional a una función delta y la parte imaginaria es proporcional a su derivada (Caldeira y Leggett 1983); así, en la versión discreta, $\eta_{k k^{\prime}}=$ 0 para $\left|k-k^{\prime}\right|>1$. Por otro lado, en el límite opuesto (adiabático), $\alpha(t)$ es constante, lo que indica un rango infinito en el tiempo para las correlaciones entre las posiciones del sistema en diferentes pasos de tiempo. Pensando en lo que ocurre entre estos dos límites, Makri y Makarov $(1994,1995)$ propusieron un algoritmo eficiente de multiplicación tensorial, en el que se emplea un tiempo límite $t_{\max }$ para el rango de memoria; así, $\eta_{k k^{\prime}} \rightarrow 0$ para $\mid k-$ $k^{\prime} \mid>\delta k_{\text {max }}$, donde $\delta k_{\text {max }} \delta t=t_{\text {max }}$, y $\delta k_{\text {max }}$ denota el número necesario de pasos en el tiempo para expandir la no-localidad temporal del funcional. La expresión analítica explícita de los términos $\eta_{k k^{\prime}}$ 's, incorporados en los cálculos numéricos de la siguiente sección, puede encontrarse en (Makri y Makarov 1994, 1995).

Para tener una discretización efectiva de las integrales sobre las variables del sistema, se utiliza la denominada representación de variable discreta (DVR-discrete variable representation; Harris, Engerholm y Gwinn 1965):

$$
\left\langle s_{i}|Q| s_{j}\right\rangle=s_{i} \delta_{i j}
$$

El esquema de multiplicación tensorial donde se desarrolla este método requiere reescribir el funcional de influencia como productos de términos correspondientes a distintos $\delta k$ :

$$
I=\prod_{k=0}^{M} \prod_{\delta k=0}^{k} I_{\delta k}\left(s_{k}, s_{k-\delta k}\right)
$$

donde $s_{k}$ se corresponde a la pareja de estados $\left(s_{k}^{+}, s_{k}^{-}\right)$, y donde cada $I_{\delta k}$, escrito en la nueva base discreta, está dado por

$$
\begin{array}{r}
I_{\delta k}\left(s_{k}, s_{k-\delta k}\right)=\exp \left[-\left(s_{k}^{+}-s_{k}^{-}\right)\right. \\
\left.\left(\eta_{k(k-\delta k)} s_{k-\delta k}^{+}-\eta_{k(k-\delta k)}^{*} s_{k-\delta k}^{-}\right)\right] .
\end{array}
$$

Así, usando la notación

$$
\left\langle s_{i}^{+}\left|e^{-\mathrm{i} H_{s} \delta t}\right| s_{j}^{+}\right\rangle\left\langle s_{j}^{-}\left|e^{\mathrm{i} H_{s} \delta t}\right| s_{i}^{-}\right\rangle=A_{0}\left(s_{i}, s_{j}\right)
$$

para el propagador libre, el esquema multiplicativo para la matriz densidad reducida queda escrita como:

$$
\begin{aligned}
& \rho\left(s^{\prime \prime}, s^{\prime} ; t=M \delta t\right)= \\
& \sum_{s_{0}, \ldots, s_{M-1}} A_{0}\left(s_{M}, s_{M-1}\right) \prod_{\delta k=0}^{M} I_{\delta k}\left(s_{M}, s_{M-\delta k}\right) \\
& \times A_{0}\left(s_{M-1}, s_{M-2}\right) \prod_{\delta k=0}^{M-1} I_{\delta k}\left(s_{M-1}, s_{M-1-\delta k}\right) \\
& \ldots \\
& \times I_{0}\left(s_{0}, s_{0}\right) \rho_{0}(0),
\end{aligned}
$$

donde $\rho_{0}(0)=\left\langle s_{0}^{+}|\rho(0)| s_{0}^{-}\right\rangle$. Esto resuelve el problema numérico de calcular la dinámica disipativa del registrador tanto en el régimen Markoviano como en el no Markoviano.

\section{Dinámica disipativa de qubits interactuantes}

Se reportó el cálculo de la dinámica cuántica para los casos de i) un qubit efectivo y de ii) dos qubits interactuantes, acoplados a un reservorio bosónico, que en general puede ser 'lento' o 'rápido' (Weiss 2008), dependiendo de las características de los modos de vibración del baño. Debido a la complejidad del método QUAPI, no ha sido posible derivar expresiones analíticas para la dinámica disipativa del entrelazamiento de qubits y los resultados aquí presentados son numéricos.

Qubit efectivo acoplado a un baño bosónico: El primer caso considerado corresponde a un sistema bipartito que se acopla bilinealmente a un baño de osciladores armónicos. Dos sistemas físicos que pueden ser descritos de esta manera pueden ser un par de puntos cuánticos acoplados entre si, en contacto con un reservorio de estado sólido (baño de fonones), donde es posible la transferencia resonante excitónica (Reina et al. 2000, Lovett et al. 2003), y pares de cromóforos (biomoléculas) en un solvente 
proteínico (Thorwart et al. 2009, Eckel et al. 2009). En estas descripciones, los cromóforos o puntos cuánticos son modelados como sistemas de dos niveles con energías de transición $E_{i}$. Para tales casos, se utiliza el modelo espín-bosón (Weiss 2008), y el Hamiltoniano del sistema acoplado puede mapearse en el de un qubit efectivo como (Eckel et al. 2009):

$$
\begin{array}{r}
H_{S}=\frac{\epsilon}{2} \sigma_{z}+\frac{\Delta}{2} \sigma_{x}+\sigma_{z} \sum_{i} g_{i}\left(a_{i}^{\dagger}+a_{i}\right) \\
+\sum_{i} \omega_{i} a_{i}^{\dagger} a_{i}
\end{array}
$$

donde $\epsilon$ es la diferencia en la energía de transición entre los qubits de cada par, y $\Delta$ denota la intensidad de la interacción entre qubits. $a_{i}$ y $a_{i}^{\dagger}$ son los operadores del baño bosónico con energía $\omega_{i}$ para el $i$-ésimo modo de vibración. Sin pérdida de generalidad, en lo que sigue se considera que cada pareja está conformada por qubits identicos $(\epsilon=0)$.

Se considera una función de densidad espectral Ohmica $J(\omega)=2 \alpha \omega \exp \left(\omega / \omega_{c}\right)$ (Weiss 2008), donde $\alpha$ es el parámetro que dá la fuerza de amortiguamiento sobre la dinámica del sistema y $\omega_{c}$ es la frecuencia de corte del reservorio (típicamente, $\omega_{c} \gg \Delta$ en el límite Markoviano).

Convergencia del Método: La convergencia del método (Figura (1) en términos del parámetro $\delta k_{\text {max }}$, donde se ha escogido el intervalo $\delta t \sim 0,1$ para cada paso en el tiempo, y en el eje vertical se ha graficado el valor esperado del operador $\sigma_{z}$, el cual representa la inversión de población entre los dos estados del sistema. Así, la Figura 10 permite escoger un valor adecuado para $\delta k_{\max }$ con el fin de optimizar el tiempo de cómputo que se requiere para cada simulación; nótese, por ejemplo, que las curvas correspondientes a $\delta k_{\max }=9$ y $\delta k_{\max }=$ 12 están prácticamente superpuestas entre sí, sin embargo, el tiempo requerido para obtener cada una de ellas, en simulaciones separadas, puede cambiar considerablemente. Así, una vez se ha garantizado la convergencia del método, se procede a realizar las simulaciones con el valor de $\delta k_{\max }$ que implique el menor costo de tiempo computacional y que no produzca perdida de la información relevante en las coherencias y poblaciones (en este caso $\delta k_{\text {max }}=9$ ).

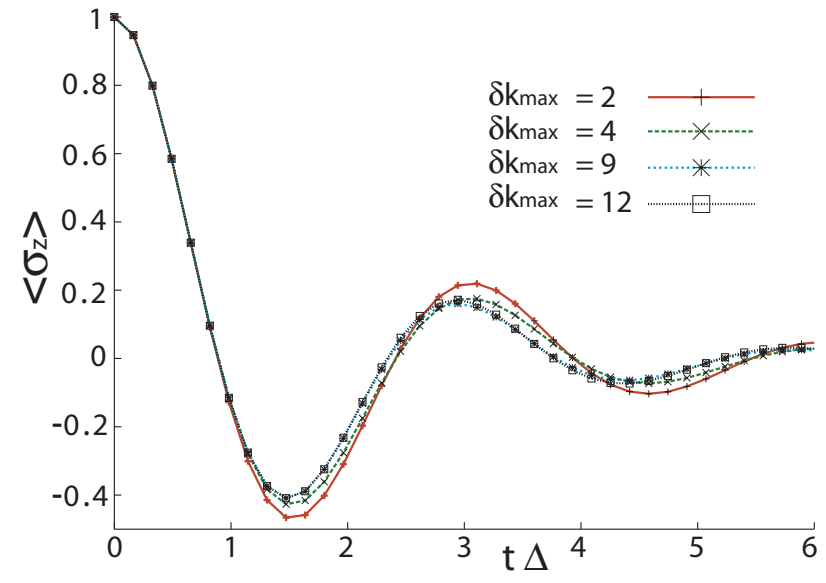

Fig. 1. Inversión de población. Sensibilidad del método para varios valores del parámetro numérico $\delta k_{\text {max }} . T=0,625 \Delta, \omega_{c}=2,5 \Delta, \alpha=$ 0,32 . Los parámetros físicos han sido tomados para comparación directa con los resultados de (Mak y Chandler 1991).

Inversión de Población: Se presenta el cálculo de la influencia de la constante de acoplamiento qubit-baño, $\alpha$, en el régimen de acoplamiento fuerte $(\alpha \sim 1)$, donde son importantes los efectos no Markovianos (Figura 2).

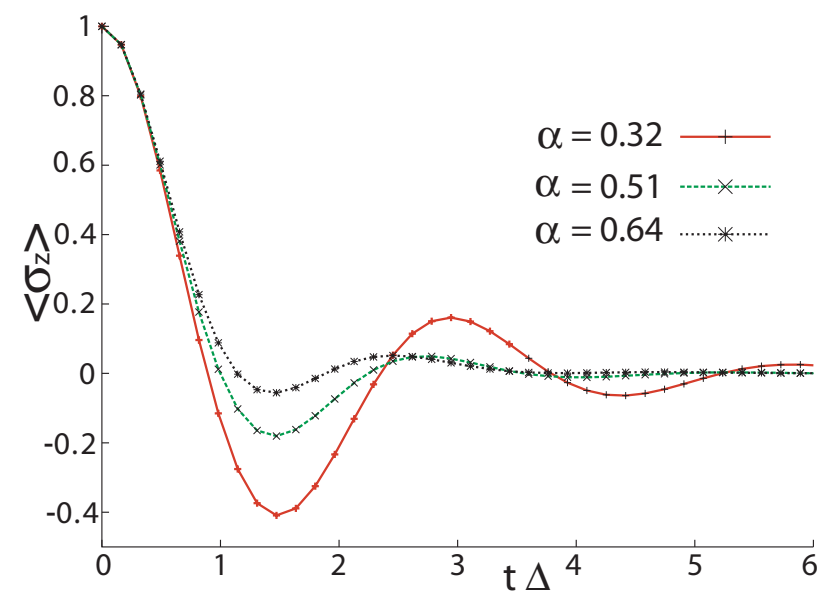

Fig. 2. Inversión de población. Comparación para diferentes valores de la constante de amortiguamiento $\alpha$. Parámetros como en la Figura 更, $\operatorname{con} \delta k_{\max }=9$.

En el caso de acoplamiento débil, $\alpha \ll 1$. Como se muestra en la Figura \2, las oscilaciones presentes en la inversión de población del sistema efectivo de dos niveles se amortiguan más rápidamente a medida que $\alpha$ crece, indicando un acoplamiento más fuerte entre el qubit y su entorno, y la consecuente pérdida en la amplitud de las oscilaciones en las poblaciones. 
Qubits interactuantes acoplados a un baño bosónico: En el segundo caso se trata dos qubits efectivos interactuantes, interacción descrita mediante un Hamiltoniano de la forma $H_{12}$, Ec. (II). Este Hamiltoniano pone de manifiesto varios tipos de acoplamiento, los cuales pueden ser modelados con la metodología aquí descrita. Se considera inicialmente un tipo de acople transversal isotrópico, es decir, $J_{x}=J_{y}=J$ y $J_{z}=0$. Este tipo de acople toma lugar en sistemas físicos de gran relevancia en el marco del procesamiento de información y cómputo cuántico, en la forma de qubits superconductores (Nielsen y Chuang 2000) y de moléculas artificiales, tales como puntos cuánticos (Reina et al. 2000, Lovett et al. 2003) y polímeros $\pi$-conjugados (Weiss 2008). El proceso físico asociado se traduce en la transferencia resonante de energía entre qubits, con intensidad de interacción $J$. El Hamiltoniano total del sistema de qubits interactuantes $H_{S T}=H_{S}^{1} \otimes$ $I_{2}+I_{1} \otimes H_{S}^{2}+H_{12}$, donde $I_{i}$ denota la matriz identidad asociada al qubit $i$, y $H_{S}^{i}$ corresponde a los términos de partícula libre (primera línea de la Ec. (3))). La matriz densidad de los qubits es descrita en la base computacional $\{|00\rangle,|01\rangle,|10\rangle,|11\rangle\}$, donde $\left|O_{i}\right\rangle\left(\left|1_{i}\right\rangle\right)$ denota el estado base (excitado) del qubit $i$, de modo que el operador densidad asociado al registrador cuántico tiene componentes $\rho_{i j, k l}$.

Poblaciones y Coberencias: La forma en que los modos de oscilación del baño afectan la dinámica cuántica del registrador se pone explícitamente de manifiesto en la Figura 3, donde se ha graficado dos de las poblaciones del sistema, $\rho_{00,00}$ y $\rho_{01,01}$ para el caso de un baño 'rápido' $\left(\omega_{c}=10 \Delta\right)$ y de uno 'lento' $\left(\omega_{c}=\Delta\right)$. Por la simetría del Hamiltoniano, las otras dos poblaciones cumplen $\rho_{11,11}=\rho_{00,00} \mathrm{y}$ $\rho_{10,10}=\rho_{01,01}$.

Es claro que un baño rápido no favorece ni la amplitud ni el tiempo de duración de las oscilaciones en las poblaciones, comparado con el caso $\omega_{c}=$ $\Delta$, donde las oscilaciones perduran por un tiempo mucho más prolongado al igual que la amplitud de las mismas.

Nótese que el parámetro $\alpha$ indica un régimen de acoplamiento fuerte para la dinámica aquí simulada. Así, es claro que, dentro del presente contexto, el baño en el régimen no-Markoviano favorece una dinámica cuántica coherente en el sistema de qubits, en contraste con lo observado para el caso del baño en el régimen Markoviano. Este hecho permitiría que operaciones de compuertas lógicas cuánticas se puedan simular en el laboratorio con una mayor fidelidad para el caso de reservorios 'lentos' (Nielsen y Chuang 2000).

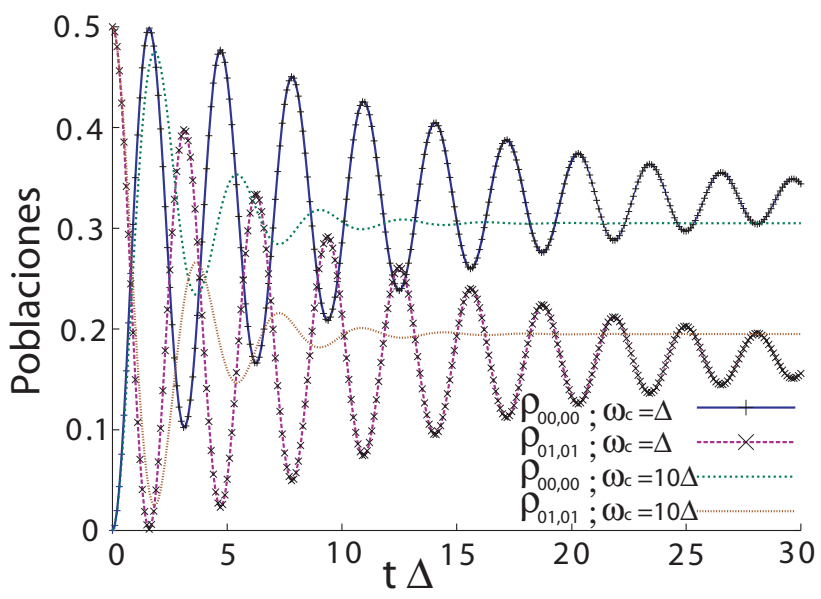

Fig. 3. Dinámica de poblaciones para $\omega_{c}=\Delta \mathrm{y}$ $\omega_{c}=10 \Delta . T=0,1 \Delta, \alpha=0,1$ y $J=0,1 \Delta$. Estado inicial $\left|\Phi_{0}\right\rangle=\frac{1}{\sqrt{2}}(|01\rangle+|10\rangle)$.

La Figura 4 muestra la dinámica de las coherencias $\rho_{01,10}+\rho_{10,01}$ y $\rho_{11,00}+\rho_{00,11}$, para $\alpha=0,1, y$ dos valores de frecuencia de corte: $\omega_{c}=\Delta$ (linea azul continua y morada a trazos largos, respectivamente), y $\omega_{c}=10 \Delta$ (linea naranja de trazo fino y linea verde de trazo intermedio, respectivamente), en el caso en el cual el registrador es preparado inicialmente en el estado $\left|\Phi_{0}\right\rangle=$ $\frac{1}{\sqrt{2}}(|01\rangle+|10\rangle)$, para los mismos parámetros de la Figura [3. A pesar de que el tipo de oscilaciones tiene sentido físico diferente, la escala de tiempo y el número de oscilaciones es similar a aquel obtenido para las poblaciones, y se marca el contraste entre los dos tipos de dinámica, dependiendo del valor considerado para $\omega_{c}$. Lo anterior se corrobora en la Figura 5, donde hemos calculado la dinámica de las coherencias $\rho_{01,10}+\rho_{10,01}$ para los casos $\omega_{c}=\Delta$ y $\omega_{c}=50 \Delta$, este último caracterizando un baño aún más rápido que el anteriormente considerado. En este caso se ha relajado un poco la constante de acoplamiento a $\alpha=0,01$. Como en el caso de la Figura 4 , es claro que los efectos de interferencia cuántica bipartita se ven favorecidos por el baño con 
$\omega_{c}=\Delta$.

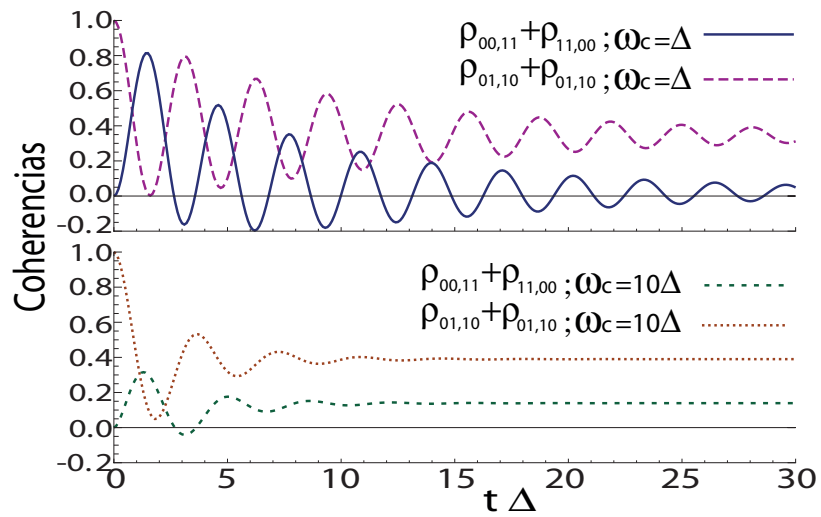

Fig. 4. Dinámica de las coherencias $\rho_{01,10}+\rho_{10,01}$ y $\rho_{11,00}+\rho_{00,11}$ para $\omega_{c}=\Delta$, y $10 \Delta . T=0,1 \Delta$, $\alpha=0,1$ y $J=0,1 \Delta$.

La Figura 5 muestra la dinámica de la coherencia de mayor importancia para la interacción transversal $J_{x}=J_{y}=J, \rho_{01,10}+\rho_{10,01}$, comparando los regímenes Markoviano y no-Markoviano generados por las características propias del baño $\left(\omega_{c}\right)$. Notese también que en este caso, debido a la reducción en la intensidad del acoplamiento sistema-reservorio $(\alpha=0,01)$, los tiempos de coherencia han sido prolongados por lo menos 5 veces que aquellos asociados al caso en el cual $\alpha=0,1$ (Figura 4 ).

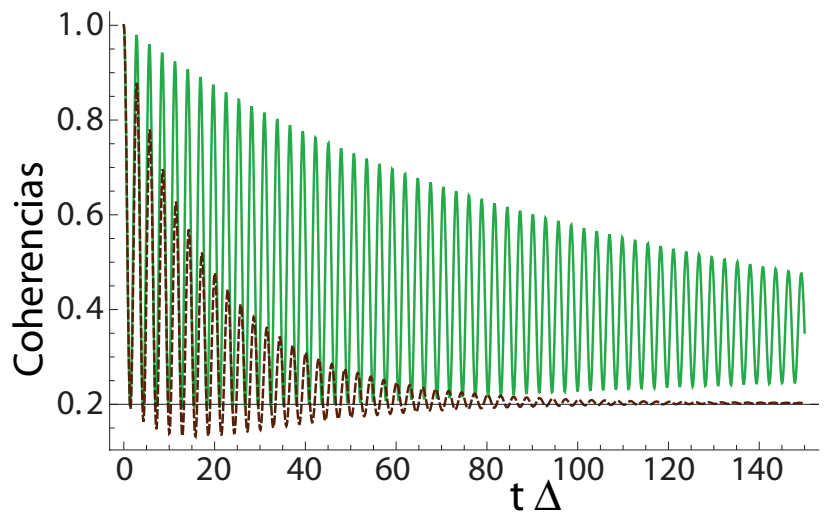

Fig. 5. Dinámica de coherencias para $\omega_{c}=\Delta$ (linea continua) y $\omega_{c}=50 \Delta$ (linea a trazos). $T=$ $0,1 \Delta, \alpha=0,01$ y $J=0,1 \Delta .\left|\Phi_{0}\right\rangle=\frac{1}{\sqrt{2}}(|01\rangle+|10\rangle)$.

Es claro entonces que las coherencias decaen mucho más rápidamente cuando el registrador se encuentra en contacto con un reservorio Markoviano y que la amplitud de las oscilaciones coherentes se vé menos afectada, o mejor, es coherentemente favorecida por un baño lento (no-Markoviano). Este mecanismo de respuesta dinámica se refleja también en el grado de correlaciones cuánticas no locales entre los qubits.
Cuantificación del entrelazamiento cuántico: Las correlaciones cuánticas no locales son cuantificadas por medio del entrelazamiento, usando la negatividad $N=\operatorname{máx}\{0,-2 \lambda\}$, siendo $\lambda$ el valor propio más pequeño de la transpuesta parcial de la matriz densidad dada por $\rho_{i j, k l}^{T_{1}}=\rho_{k j, i l}$ (Peres, 1996; Horodecki, Horodecki y Horodecki, 1996). Para una constante de interacción qubit-qubit $J=0,1 \Delta$, un ruido cuántico no-Markoviano $\left(\omega_{c}=\Delta\right)$ ayuda a que el entrelazamiento persista por un tiempo más prolongado en el registrador cuántico, en contraste con el caso de un ruido Markoviano, donde el entrelazamiento se suprime completamente en un tiempo mucho más corto (menos de $1 / 3$ del tiempo anterior; Figura (6).

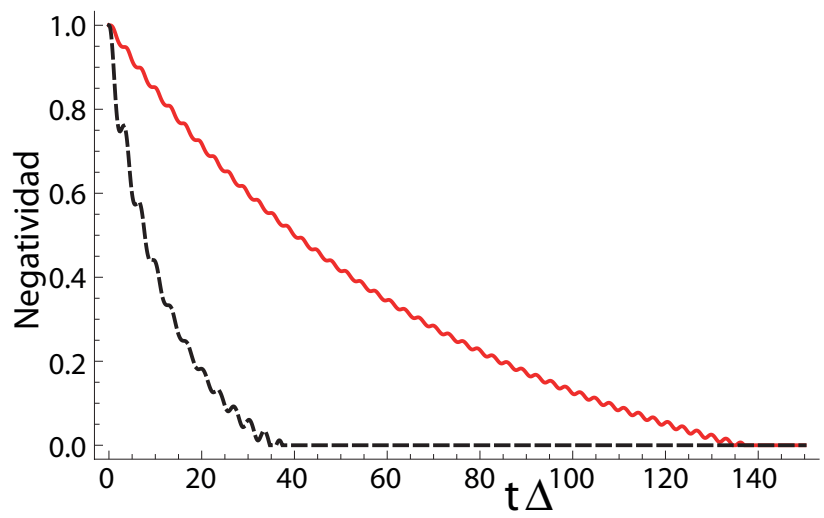

Fig. 6. Dinámica de entrelazamiento para $\omega_{c}=\Delta$ (linea roja continua ), y $\omega_{c}=50 \Delta$ (linea negra a trazos). $T=0,1 \Delta, \alpha=0,01$ y $J=0,1 \Delta .\left|\Phi_{0}\right\rangle=$ $\frac{1}{\sqrt{2}}(|01\rangle+|10\rangle)$.

Un comportamiento similar puede ser observado para una interacción más fuerte entre los qubits (Figura Z), $J=\Delta$; en este caso el entrelazamiento presenta oscilaciones más pronunciadas que se deben a la interferencia constructiva que dicho acople introduce en la dinámica disipativa.

De la dinámica del entrelazamiento calculada, resulta razonable asumir para la negatividad un comportamiento de la forma

$$
N(t)=e^{-\Gamma t}+A_{\infty}
$$

en el caso en que el sistema comience en un estado máximamente entrelazado $(N(0)=1)$, donde la rata de decaimiento $\Gamma$ indica qué tan rápido decae el entrelazamiento, y $A_{\infty}$ indica si el decaimiento es asintótico a cero, o si hay presencia del fenómeno de 
desvanecimiento súbito de entrelazamiento (ESD).

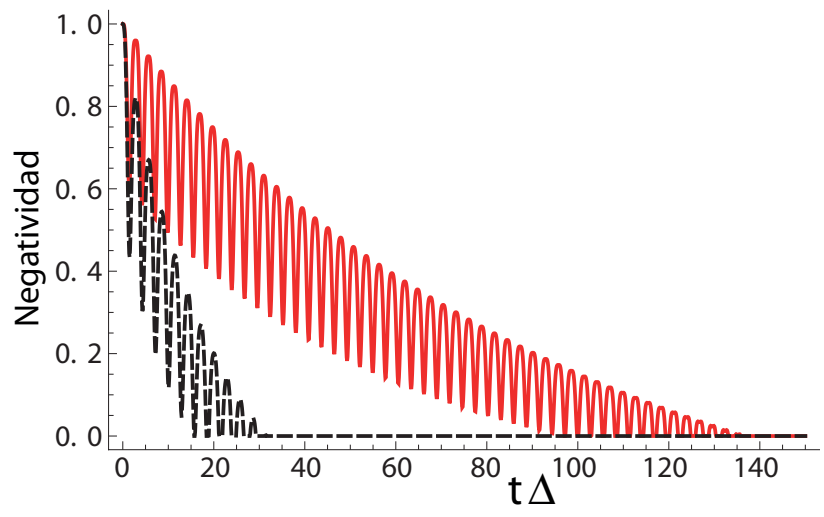

Fig. 7. Dinámica de entrelazamiento para $\omega_{c}=\Delta$ (linea roja continua ), y $\omega_{c}=50 \Delta$ (linea negra cortada). $J=\Delta$. Demás parámetros como en la Figura 6 .

De la Figura 6 es posible deducir que estas dos cantidades dependen fuertemente de la frecuencia de corte $\omega_{c}$, indicando la importancia del régimen (dígase Markoviano o no Markoviano) de actuación del ruido sobre el registrador. Nótese que la razón de decaimiento se incrementa drásticamente cuando aumenta la frecuencia de corte, y que el tiempo para el cual el entrelazamiento se hace cero cambia abruptamente con dicha frecuencia, comportamiento funcional que tiene el parámetro $A_{\infty} \operatorname{con} \omega_{c}$.

Aunque la negatividad presenta oscilaciones de amplitud notablemente mayor al incrementar la intensidad de interacción $J$ (Figura Z), se puede inferir que una función de la forma dada por la Ec. (4) describe el comportamiento envolvente de esta cantidad, y que el análisis hecho antes para la Figura 6 tiene lugar. Sin embargo, la forma de la función con la que se describe el entrelazamiento puede ser más compleja que la mostrada por la Ec. (4). Existe un comportamiento aún más interesante que puede observarse en la dinámica de la negatividad, esto es, un comportamiento de colapsos y resurgimientos sucesivos. Este puede describirse funcionalmente de la forma

$$
N(t)=e^{-\Gamma t} \cos (\Omega t+\phi)+A_{\infty},
$$

donde ahora la función $\cos (\Omega t+\phi)$ permite que para ciertos intervalos, el valor de $N(t)$ sea cero, dependiendo del valor que tome la constante $A_{\infty}$. En este contexto, $\Omega$ y $\phi$ denotan la frecuencia de oscilación del entrelazamiento y su fase, respectivamente. Se muestra como el entrelazamiento decae a cero por intervalos cortos y posteriormente vuelve a crecer (Figura 8); claramente este comportamiento es obtenido en el régimen puramente no Markoviano de la dinámica del sistema-baño, $\omega_{c}=0,5 \Delta$. Tal comportamiento no es observado en el caso de acoplamiento a un baño rápido.

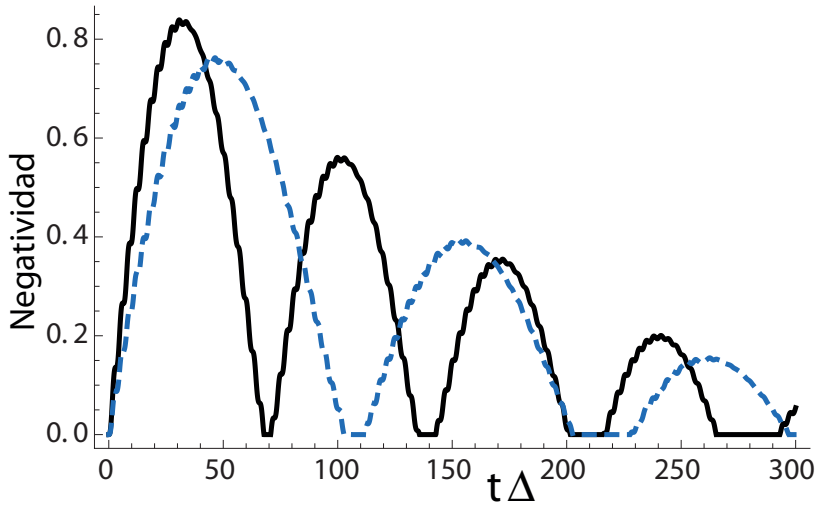

Fig. 8. Dinámica de entrelazamiento para $J_{x}=$ $J_{y}=0,1 \Delta, J_{z}=0$ (linea negra continua ), y $J_{x}=$ $J_{y}=0, J_{z}=0,1 \Delta$ (linea azul a trazos); $\omega_{c}=0,5 \Delta$. Demás parámetros como en la Figura 6 , excepto que ahora $\left|\Phi_{0}\right\rangle=|11\rangle$.

El tipo de interacción presente entre los qubits es de crucial importancia en la dinámica de entrelazamiento. De la Figura $\varangle$ se observa que al cambiar de una interacción de tipo transversal $J_{x}=$ $J_{y}=J=0,1 \Delta, J_{z}=0$, a una de tipo longitudinal $J_{x}=$ $J_{y}=0, J_{z}=0,1 \Delta$, los tiempos para los cuales ocurren los colapsos y resurgimientos, así como la amplitud en las oscilaciones del entrelazamiento, cambian drásticamente, lo que implica que en la Ec. (5), los valores de las constantes de decaimiento y frecuencia de oscilación cambian notoriamente de acuerdo a la escogencia de los $J_{i}$. Físicamente, el caso de 'interacción longitudinal' podría estar representado por la interacción entre espines electrónicos en puntos cuánticos (Benjamin, Lovett y Reina 2004). Finalmente, es importante anotar los tiempos extremadamente largos para los cuales persiste el entrelazamiento, dadas las condiciones consideradas en la Figura 8 .

\section{Conclusión}

La dinámica de entrelazamiento para un sistema de qubits interactuantes, acoplados a un reservorio de modos bosónicos caracterizado por una frecuencia de corte $\omega_{c}$, se calculó de manera exacta haciendo uso del método numérico exacto QUAPI. Se encontró que la dinámica cuántica coherente es asistida, favorecida y prolongada en el tiempo para el caso de una interacción sistema-baño 
en el régimen estrictamente no Markoviano y que un baño "lento" favorece el proceso de coherencia e interferencia cuántica en el registrador. Dentro de esta descripción, dos comportamientos crucialmente diferentes en la fenomenología del entrelazamiento se encontrarón: el desvanecimiento súbito de entrelazamiento y los colapsos y resurgimientos del mismo. Tales características fueron mapeadas dentro de una dependencia funcional que permite identificar y cuantificar con claridad ambos comportamientos. Tal dependencia es altamente sensible a las condiciones iniciales y a la frecuencia de corte, así como también al factor de acoplamiento sistema-baño $\alpha$, y a la intensidad de interacción entre qubits $J$.

A la luz de los avances teórico-experimentales sobre teoría de información y de cuantificación de correlaciones en sistema cuánticos, se plantean nuevos horizontes de investigación. En particular, el estudio de otros tipos de correlaciones, más allá del entrelazamiento, y probablemente más generales y fundamentales (Ollivier y Zurek 2001, $\mathrm{Xu}, \mathrm{Xu}, \mathrm{Li}$, Zhang, Zou y Guo 2010) son de relevancia actual, así como sus posibles aplicaciones a sistemas cuánticos de gran pertinencia como son los sistemas de moléculas individuales acopladas (Susa y Reina 2012), los complejos fotosintéticos biomoleculares (Thorwart et al. 2009, Eckel et al. 2009) y otros sistemas nano-estructurados, ya sea natural o artificialmente.

\section{Agradecimientos}

A la Vicerrectoría de Investigaciones de la Universidad del Valle por la financiación parcial a través del Proyecto No. CI 7859. C. E. Susa agradece al programa de Colciencias Jovenes Investigadores $e$ Innovadores Virginia Gutiérrez de Pineda.

\section{Conflicto de intereses}

Este trabajo no presenta conflicto de intereses.

\section{Referencias}

Almeida MP, de Melo F, Hor-Meyll M, Salles A, Walborn SP, Souto Ribeiro PH, Davidovich L (2007) Environment-induced sudden death of entanglement. Science 316:579-582

Bellomo B, Lo Franco R, Compagno G, (2007) Non-Markovian effects on the dynamics of entanglement. Phys. Rev. Lett. 99:160502/1-4

Benjamin SC, Lovett B, Reina JH (2004)
Optical quantum computation with perpetually coupled spins. Phys. Rev. A 70:060305(R)/1-4

Bennett CH, Brassard G, Crépeau C, Jozsa R, Peres A, Wootters WK (1993) Teleporting an unknown quantum state via dual classical and Einstein-Podolsky-Rosen channels. Phys. Rev. Lett. 70:1895-1899

Bennett CH, DiVincenzo D (2000) Quantum information and computation. Nature 404:247-255

Caldeira AO, Leggett AJ (1983) Path integral approach to quantum Brownian motion. Physica A 121:587-616

Cummings NI, $\mathrm{Hu}$ BL (2008) Dynamics of atom-field entanglement: Towards strong-coupling and non-Markovian regimes. Phys. Rev. A 77:053823/1-12

Dodd PJ, Halliwell JJ (2004) Disentanglement and decoherence by open system dynamics. Phys. Rev. A 69:052105/1-6

Eckel J, Reina JH, Thorwart M (2009) Coherent control of an effective two-level system in a non-Markovian biomolecular environment. New Journal of Physics 11:085001/1-18

Fanchini FF, Cornelio MF, de Oliveira MC, Caldeira AO (2011) Conservation law for distributed entanglement of formation and quantum discord. Phys. Rev. A. 84:012313/1-4

Feynman RP, Vernon FL (1963) The theory of a general quantum system interacting with a linear dissipative system. Journal Annals of Physics 24:118-173

Ficek Z, Tanás R (2002) Entangled states and collective nonclassical effects in two-atom systems. Physics Reports 372:369-443

Ficek Z, Tanás R (2008) Delayed sudden birth of entanglement. Phys. Rev. A. 77:054301/1-4

Gardiner CW, Zoller P (2004) Quantum Noise. Springer Series in Synergetics, Springer

Gisin N, Ribordy G, Tittel W, Zbinden H (2002) Quantum cryptography. Rev. Mod. Phys. 74:145-195

Hammerer K, Vidal G, Cirac JI (2002) Characterization of nonlocal gates. Phys. Rev. A 66:062321/1-13 
Harris DO, Engerholm GG, Gwinn WD (1965) Calculation of matrix elements for one dimensional quantum-mechanical problems and the application to anharmonic oscillators. J. Chem. Phys. 43:1515-1517

Horodecki M, Horodecki P, Horodecki R (1996) Separability of mixed states: necessary and sufficient conditions. Phys. Lett. A 223:1-8

Konrad T, de Melo F, Tiersch M, Kasztelan C, Aragão A, Buchleitner A (2008) Evolution equation for quantum entanglement. Nature Physics 4:99-102

Ladd TD, Jelezko F, Laflamme R, Nakamura Y, Monroe C, O'Brien JL (2010) Quantum computers. Nature 464:45-53

Lovett B, Reina JH, Nazir A, Briggs A (2003) Optical schemes for quantum computation in quantum dot molecules. Phys. Rev. B 68:205319/1-18

Mak CH, Chandler D (1991) Coherent-incoherent transition and relaxation in condensed-phase tunneling systems. Phys. Rev. A. 44:2352-2369

Makri N, Makarov D (1994) Path integrals for dissipative systems by tensor multiplication. Condensed phase quantum dynamics for arbitrarily long time. Chem. Phys. Lett. 221:482-491

Makri N, Makarov D (1995) Tensor propagator for iterative quantum time evolution of reduced density matrices. I. Theory. J. Chem. Phys. 102:4600-4610

Mermin ND (1985) Is the moon there when nobody looks? Reality and quantum theory. Physics Today 38:38-47

Mintert F, Carvalho AR, Kus M, Buchleitner A (2005) Measures and dynamics of entangled states. Phys. Rep. 415:207-259

Nielsen MA Chuang IL (2000) Quantum Computation and Quantum Information. Cambridge. Cambridge University Press

Ollivier H, Zurek WH (2001) Quantum discord: A measure of the quantumness of correlations. Phys. Rev. Lett. 88:017901/1-4

Peres A (1996) Separability criterion for density matrices. Phys. Rev. Lett. 77:1413-1415
Petterson J, Bailey B (2010) Solid State Physics: Introduction of the theory. Springer. Springer-Verlag Berlin

Quiroga L, Rodriguez FJ, Ramírez ME, París R (2007) Nonequilibrium thermal entanglement. Phys. Rev. A 75:032308/1-5

Reina JH, Quiroga L, Johnson N (2000) Quantum entanglement and information processing via excitons in optically driven quantum dots. Phys. Rev. A 62:012305/1-8

Scala M, Migliore R, Messina A, Sánchez-Soto LL (2011) Robust stationary entanglement of two coupled qubits in independent environments. Eur. Phys. J. D 61:199-205

Shresta S, Anastopoulos C, Dragulescu A, Hu BL (2005) Non-Markovian qubit dynamics in a thermal field bath: Relaxation, decoherence, and entanglement. Phys. Rev. A 71:022109/1-10

Susa CE Reina JH (2010) Nonlocal Fluctuations and control of dimer entanglement dynamics. Phys. Rev. A 82:042102/1-9

Susa CE, Reina JH (2012) Correlations in optically controlled quantum emitters. Phys. Rev. A 85:022111/1-10

Thorwart M, Eckel J, Reina JH, Nalbach P, Weiss $S$ (2009) Enhanced quantum entanglement in the non-Markovian dynamics of biomolecular excitons. Chem. Phys. Lett. 478:234-237

Weiss U (2008) Quantum Dissipative Systems, World Scientific, Singapore

Xu J-S, Xu X-Y, Li Ch-F, Zhang Ch-J, Zou X-B, Guo G-C (2010) Experimental investigation of classical and quantum correlations under decoherence. Nature Comm. 1:7/1-6; doi: $10.1038 /$ ncomms1005

Yu T, Eberly JH (2003) Qubit disentanglement and decoherence via dephasing. Phys. Rev. B 68:165322/1-9;

Yu T, Eberly JH (2004) Finite-time disentanglement via spontaneous emission. Phys. Rev. Lett. 93:140404/1-4;

Yu T, Eberly JH (2006) Quantum open system theory: bipartite aspects Phys. Rev. Lett. 97:140403/1-4

Yu T, Eberly JH (2009) Sudden death of entanglement. Science 323:598-601 


\section{Coherencia y entrelazamiento en dinámica no Markoviana de qubits}

Resumen. Se presenta el análisis de la dinámica de entrelazamiento de un sistema bipartito de bits cuánticos (qubits) en el régimen no Markoviano. En este, la escala temporal en la cual evolucionan los grados de libertad del reservorio es del mismo orden de magnitud (o menor) que aquella correspondiente a la evolución del sistema de qubits. En la descripción de la evolución cuántica de los qubits en el régimen no Markoviano se utiliza el método numérico exacto de la Integral de Caminos Cuasi Adiabática-QUAPI. Se calculó la dinámica del operador densidad de los qubits, a partir de la cual se cuantificó la dinámica de las coherencias y de las poblaciones y la influencia del ruido cuántico sobre los qubits. Utilizando la negatividad como métrica, se calculó la dinámica de correlaciones cuánticas no locales (entrelazamiento), la cual permitió identificar una estructura funcional asociada al régimen no Markoviano en la cual se destacan el desvanecimiento súbito y el colapso y el resurgimiento del entrelazamiento.

Palabras-Clave: Qubits; informacion cuántica; entrelazamiento; decoherencia no Markoviana; nanoestructuras.

\section{Coerência e emaranhamento em dinâmica não-Markoviana de qubits}

Resumo. Apresentamos a análise da dinâmica do emaranhamento num sistema de bits quânticos (qubits) no regime não-Markoviano. Em tal regime, a escala de tempo em que os graus de liberdade do reservatório evolua, é da mesma ordem de magnitude (ou inferior) ao que corresponde à evolução do sistema de qubits. Para fazer isso, nós desenvolvemos o método numérico exato conhecido como a abordagem da Integral do Caminho Quasi-adiabático-QUAPI, a fim de descrever a dinâmica dissipativa dos qubits em tal regime não-Markoviano. Isto permitiu-nos calcular a dinâmica do operador densidade dos qubits, a partir do qual foi quantificada a dinâmica das coerências e populaçẽs e a influência do ruído quântico nos qubits. Usando a negatividade como uma métrica, nós calculamos a dinâmica de correlações quânticas não-locais (emaranhamento), permitindo-nos interpretar os fenômenos quânticos não-Markovianos em termos da morte súbita e do colapso e renascimento de emaranhamento.

Palavras-Chave: Qubits; informação quântica; emaranhamento; decoerência não-Markoviana; nanoestruturas. 